\title{
Phenolic film engineering for template-mediated microcapsule preparation
}

Hirotaka Ejima $^{1,2}$, Joseph J. Richardson ${ }^{2}$ and Frank Caruso ${ }^{2 *}$

${ }^{1}$ Institute of Industrial Science, The University of Tokyo, 4-6-1 Komaba, Meguro-ku, Tokyo 153-8505, Japan

${ }^{2}$ Department of Chemical and Biomolecular Engineering, The University of Melbourne, Parkville, Victoria 3010, Australia

*Tel: +61-3-8344-3461, Fax: +61-3-8344-4153, E-mail: fcaruso@unimelb.edu.au.

Keywords: polyphenol; drug delivery system; microcapsule; pH-responsive film; bio-based polymer 


\begin{abstract}
Microcapsules are of scientific and technological interest because of their ability to encapsulate cargo inside their hollow interiors, thereby separating and protecting the cargo from the external environment. Both template-free and -mediated strategies have been exploited to prepare microcapsules. For the latter strategy, coating sacrificial particulate templates with robust films is a key step towards obtaining mechanically-stable hollow architectures following template removal. In this review, we focus on phenolic-based film engineering techniques utilizing dopamine (DA) and tannic acid (TA), which have recently emerged as new platforms for template-mediated capsule preparation. The first part of the review describes the selfpolymerization of DA, which preferentially occurs at interfaces. The second part of the review describes TA capsules. Particular emphasis is placed on the coordination-triggered rapid deposition of TA on different substrates. These examples highlight the versatility and simplicity of phenolic film engineering strategies for microcapsule preparation. Phenolics are abundant biobased materials, and thus form an attractive field of research for the future development of microcapsules.
\end{abstract}




\section{INTRODUCTION}

Polymer microcapsules have drawn considerable interest for various applications, especially drug delivery, since they can encapsulate cargo (e.g. therapeutics), and release them in a controllable manner. There exist two major strategies for preparing polymer microcapsules, namely template-free assembly and template-mediated assembly. ${ }^{1}$ Representative template-free methods are the self-assembly of polymersomes ${ }^{2}$ and polyion complex vesicles ${ }^{3}$ from block copolymers. In the template-mediated assembly method, which is the focus of this review, polymeric shells can be assembled via the sequential deposition of complementary polymers (layer-by-layer, LbL $^{4-11}$ ) around sacrificial template particles. ${ }^{12}$ Hollow polymer microcapsules can be obtained by selectively removing the template cores without disrupting the LbLassembled polymeric shells. Despite the significant progresses in the field of LbL-engineered microcapsules, the manufacturing process inherently involves multiple alternating deposition steps of complementary polymers, rendering it time consuming and labor intensive. To circumvent this limitation, we recently developed electrophoretic ${ }^{13}$ and immersive ${ }^{14}$ LbL techniques for simplifying and automating the capsule preparation processes. Recently, the onestep deposition of phenolic-based thin films has emerged as a rapid, simple and cheap alternative technology for template-mediated microcapsule preparation. This technique has opened up practical processing methods that researchers can readily access and explore to prepare a diverse range of functional materials.

Phenolic compounds are naturally occurring materials widely found in living organisms. ${ }^{15}$ The significant interest in phenolic compounds in recent years is attributed to their broad spectrum of chemical and biological properties. For instance, plant-derived phenolic compounds in tea and wine are major flavor determinants and are believed to have a number of 
pharmacological benefits on human health. ${ }^{16}$ Animals also take advantage of phenolics, as phenolic (L-3,4-dihydroxyphenylalanine)-containing proteins play a crucial role in the adhesive functions of mussel feet. ${ }^{17}$ From the engineering point of view, the stickiness, bio-based nature, and metal-coordination capabilities, as well as antioxidant, anticarcinogenic, and antibacterial properties of phenolic compounds, are attractive for developing biofunctional 'green' materials. ${ }^{18}$

In 2007, Lee et al. reported the formation of mussel-inspired multifunctional coatings on a wide range of substrates. ${ }^{19}$ The deposition of polydopamine (PDA) films takes place in onestep on virtually any surface by simply dipping the object of interest into an aqueous solution of dopamine (DA). Although the molecular-level mechanism is still under debate, ${ }^{20}$ recent research suggests that non-covalent self-assembly and covalent polymerization both contribute to the PDA film formation. ${ }^{21}$ Recently, we reported the coordination-triggered deposition of tannic acid (TA)-metal films on a wide range of substrates. ${ }^{22}$ This deposition process is more rapid than that of PDA, and the obtained films can be disassembled at low $\mathrm{pH}$. By depositing these phenolics on sacrificial particulate templates, hollow microcapsules with advanced functions can be assembled.

\section{PDA CAPSULES}

Postma et al. first reported the template-mediated preparation of PDA capsules in 2009 (Figure 1). ${ }^{23}$ Sacrificial particulate templates $\left(\mathrm{SiO}_{2}\right)$ were incubated in DA solution $(10 \mathrm{mM}$ tris(hydroxymethyl)-aminomethane (Tris)- $\mathrm{HCl}$ at $\mathrm{pH}$ 8.5), and the sacrificial $\mathrm{SiO}_{2}$ particles were subsequently removed by hydrofluoric acid (HF). The diameter of the sacrificial templates (0.5$5 \mu \mathrm{m}$ were used) directly translated to the size of the assembled PDA capsules. The thickness of the PDA shells varied with changing incubation time (ca. 10-20 nm). By simply repeating the 
deposition step, the thickness could be further increased in set increments. In 2009, Yu et al. also reported the preparation of PDA capsules, and examined their loading and release properties. ${ }^{24}$ Rhodamine 6G (Rh6G) and methyl orange (MO) were used as model cargo and the effect of several parameters ( $\mathrm{pH}$, capsule size, shell thickness, and solvent) on the loading and release kinetics were investigated. ${ }^{24,25}$ DA modified poly(L-glutamic acid) (PGA) has also been selfpolymerized and deposited on sacrificial particulate templates in a similar way to pure DA (Figure 2). ${ }^{26}$ The protease-triggered degradation of PGA-PDA films was achieved and quantified by quartz crystal microgravimetry (QCM).

Liu et al. demonstrated the preparation of carbon capsules via the carbonization of PDA. ${ }^{27}$ First, $\mathrm{SiO}_{2}$ particles were coated with PDA, then the PDA layer was carbonized at 800 ${ }^{\circ} \mathrm{C}$, and finally the $\mathrm{SiO}_{2}$ cores were removed by treatment with $\mathrm{HF}$ (Figure 3a,b). By starting with $\mathrm{Au@SiO} 2$ core-shell particles, ${ }^{28}$ instead of $\mathrm{SiO}_{2}$ templates, carbon capsules encapsulating single Au nanoparticles were obtained (Figure 3c). These yolk-shell carbon capsules showed high catalytic activity and good recyclability in the reduction of 4-nitrophenol into 4aminophenol.

Cui et al. showed that low-molecular-weight polydimethyldiethoxysilane (PDMS) oil-inwater emulsion droplets ${ }^{29}$ are favorable sacrificial templates for the preparation of PDA capsules, ${ }^{30}$ as these templates can be removed with ethanol ( $c f . \mathrm{SiO}_{2}$ needs to be removed with HF). Furtheremore, the diameter of these droplets can be readily tuned from hundreds of nanometers to several micrometers by changing either the condensation time or the concentration of the precursor dimethyldiethoxysilane (DMDES). In conventional LbL assembly, the template particles need to be spun down after each deposition step to remove excess polymer; however, the PDMS templates have a density lower than water and are difficult to spin down. Therefore, 
the one-step deposition process of PDA, without any need for intermittent centrifugation steps, allows the use of PDMS emulsion droplets as sacrificial templates. The advantage of oil-in-water emulsion droplets is that hydrophobic cargo, such as magnetic $\mathrm{Fe}_{3} \mathrm{O}_{4}$ nanoparticles, fluorescent quantum dots (CdSe/CdS), or anticancer drug (thiocoraline), can easily be preloaded into the emulsion prior to PDA coating (Figure 4). ${ }^{30}$ Wang et al. utilized alkane-in-water emulsions as templates for the preparation of PDA capsules, ${ }^{31}$ and it was found that the interfacial basicity of the droplets promoted the self-polymerization of DA.

PDA films can be used as the basis for surface-initiated atom-transfer radical polymerization (ATRP) ${ }^{32,33}$ Kohri et al. demonstrated the co-deposition of DA and ATRP initiator-bearing DA (DA-BiBB) onto polystyrene (PS) particles. ${ }^{32}$ 2-hydroxyethyl methacrylate (HEMA) was polymerized to give a polyHEMA brush-functionalized PDA layer. The transparency of the films increased as the DA-BiBB fraction was increased. Colorless phenolic coatings are desired in some cases, as the characteristic black color of PDA can hinder some practical application. ${ }^{18}$ The further functionallization of the polyHEMA-PDA capsules with fluorescent dansyl group was carried out in acetone in the presence of triethylamine. Ma et al. prepared dual-responsive capsules sensitive to $\mathrm{pH}$ and temperature changes using the surfaceinitiated ATRP of 2-(2-methoxyethoxy)ethyl methacrylate $\left(\mathrm{MEO}_{2} \mathrm{MA}\right)$ and oligo(ethylene glycol) methacrylate (OEGMA) on the PDA base layer (Figure 5). ${ }^{33}$ The charge of PDA is dependent on the $\mathrm{pH}$, while the poly( $\mathrm{MEO}_{2} \mathrm{MA}-\mathrm{co}$-OEGMA) has a lower critical solution temperature (LCST), which can be tuned by changing the molar fraction of OEGMA. Thus, the copolymer-functionalized PDA capsules exhibited dual-responsive loading and release of dye molecules (Rh6G and MO). 
Organic-inorganic hybrid capsules were prepared using PDA as a bioadhesive layer. ${ }^{34}$ First, the $\mathrm{CaCO}_{3}$ template particles were coated with protamine, which induced the hydrolysis and condensation of titania or silica precursor to form the second inorganic layer. These particles were finally capped with the third layer, $\mathrm{PDA}$, and the $\mathrm{CaCO}_{3}$ cores were removed with ethylenediaminetetraacetic acid (EDTA) treatment. The PDA/titania capsules exhibited superior mechanical stability than the PDA/silica due to the strong coordination interactions between $\mathrm{Ti}^{\mathrm{IV}}$ and the catecholic groups in PDA. DA-modified alginate was also used to construct LbL capsules based on the coordination with $\mathrm{Ti}^{\mathrm{IV}}{ }^{35}$

PDA capsules were also used as a vehicle for drug delivery into living cells (Figure 6). ${ }^{36}$ The anticancer drug doxorubicin (Dox) was conjugated to a thiolated poly(methacrylic acid) (PMA $\left._{\mathrm{SH}}\right)$ through acid-cleavable hydrazone bonds. The thiol functionalities on PMA $\mathrm{SH}_{\mathrm{SH}}$ were used to immobilize this drug-polymer conjugate on the PDA shells based on the Michael addition (Figure 6a). The Dox release from PDA capsules was investigated at $\mathrm{pH}$ 7.4, 6.0 and 5.0, simulating the physiological $\mathrm{pH}$ in the extracellular space, subcellular endosomes, and lysosomes, respectively. Although $85 \%$ and $40 \%$ of the encapsulated Dox was released at pH 5.0 and 6.0 over $12 \mathrm{~h}$, respectively, less than $20 \%$ was released at $\mathrm{pH}$ 7.4. Cell viability assays using a cervical cancer cell line (HeLa) showed enhanced cytotoxicity of Dox-loaded PDA capsules, compared with free Dox under the same conditions, demonstrating the effectiveness of PDA capsules for drug delivery in living cells.

\section{TA CAPSULES}

TA is a biodegradable phenolic compound of natural origin, which has a central glucose core connected with gallic acids through ester bonds at the hydroxyl groups of glucose (Figure 7,8). 
TA has a high spectrum of bioactivities such as antioxidant, ${ }^{37}$ antibacterial, ${ }^{38}$ anticarcinogenic, ${ }^{39}$ antimutagenic $^{40}$ and antiviral $^{41}$ properties. Through electrostatic or hydrogen bonding interactions, TA has been assembled via the LbL assembly method with poly(dimethyldiallylamide) (PDDA), ${ }^{42}$ poly(allylamine) (PAH), ${ }^{42-44}$ poly( $N$-vinylcaprolactam) (PVCL) ${ }^{45,46}$ poly( $N$-vinylpyrrolidone) (PVPON), ${ }^{45-47}$ poly(ethylene oxide) (PEO), ${ }^{45} \operatorname{poly}(N$ isopropylacrylamide) (PNIPAM), ${ }^{45,46}$ poly(2-n-propyl-2-oxazoline), ${ }^{48} \quad \mathrm{Fe}^{\mathrm{III}} \quad$ ions, ${ }^{49,50}$ and proteins $^{51}$ to form thin films. The first microcapsules based on TA were prepared by Shutava et al. via multistep LbL assembly in 2005 . $^{42}$

Lomas et al. used TA to construct polymersome-loaded multicompartment capsules (Figure 7). ${ }^{52}$ Polymersomes formed from poly(oligo(ethylene glycol) methacrylate)-blockpoly(2-(diisopropylamino)-ethyl methacrylate) (POEGMA-b-PDPA) were incorporated into multilayers of TA and PVPON. TA acts as an efficient hydrogen-bond donor for both PVPON and the POEGMA corona of the polymersomes. The polymersomes disassembled at low endocytic $\mathrm{pH}$ due to the $\mathrm{pH}$-dependent nature of the PDPA block (i.e., deprotonated at physiological $\mathrm{pH}$ and protonated at endocytic $\mathrm{pH}$ ), hence the polymersomes could release a therapeutic cargo in a pH-dependent manner. The polymersome-loaded capsules showed the release of a plasmid DNA encapsulated within the polymersome subcompartments in response to the lowering $\mathrm{pH}$ from physiological to endocytic conditions.

Recently, we found that the instantaneous film deposition of TA occurred on a wide range of substrates, including inorganic, organic, and biological substrates, triggered by TA complexation with $\mathrm{Fe}^{\mathrm{III}}$ ions (Figure 8). ${ }^{22}$ The formation of $\mathrm{Fe}^{\mathrm{III}}-\mathrm{TA}$ films on particulate PS templates was readily confirmed by the negative shift of the surface zeta potential relating to TA and the color change from white to dark blue relating to the complexation of Fe. After removing 
the PS templates, highly uniform microcapsules were obtained (Figure 8b-d). The shell thickness was about $10 \mathrm{~nm}$, as determined by atomic force microscopy (AFM) height analysis. Analogous to LbL assembly, the thickness could be further increased by simply repeating the coating procedure. The mechanical properties of the $\mathrm{Fe}^{\mathrm{III}}$-TA capsules were examined by AFM force measurements. Young's modulus $\left(E_{\mathrm{Y}}\right)$ of the $D=3.6 \mu \mathrm{m}$ capsules was $1.0 \pm 0.2 \mathrm{GPa}$, which is at the high end of the range observed for LbL polyelectrolyte capsules (0.01 to $1 \mathrm{GPa}){ }^{53} \mathrm{The} \mathrm{Fe}^{\mathrm{III}}$ TA capsules disassembled in a pH-sensitive manner, as coordination between $\mathrm{Fe}^{\mathrm{III}}$ and $\mathrm{TA}$ is $\mathrm{pH}-$ dependent. The competitive chelation of $\mathrm{Fe}^{\mathrm{III}}$ (using EDTA) markedly accelerated the disassembly of the $\mathrm{Fe}^{\mathrm{III}}$ - TA capsules even at neutral $\mathrm{pH}$, suggesting the main interaction between $\mathrm{Fe}^{\mathrm{III}}$ and TA is coordination bonding. It is noteworthy that the metal species chelated by TA are

not limited to $\mathrm{Fe}^{\mathrm{III}}$, as was demonstrated for other metals such as $\mathrm{V}^{\mathrm{III}}$, $\mathrm{Al}^{\mathrm{III}}$, and $\mathrm{Gd}^{\mathrm{III}}$. 22,54 Solutions of TA capsules prepared with various metals exhibited a wide spectrum of colors that depend on the metal species chelated; ${ }^{54}$ thus where color matters, a judicious choice of metal can be made depending on the specific application. For metal-phenolic network films, the simple preparation, negligible cytotoxicity, $\mathrm{pH}$-responsive disassembly profile, and extendibility to various metals all provide a platform for the engineering and assembly of promising architectures useful for biomedical applications.

\section{SUMMARY}

Recent advances in the preparation of microcapsules using phenolics have been reviewed herein. Phenolic microcapsules can be assembled via the multistep LbL assembly method or via onestep deposition methods. The sacrificial particles necessary for templating the phenolic films into microcapsules can be of varying composition (solid or liquid) allowing for the facile encapsulation of hydrophobic or hydrophilic cargo depending on the template particles used. By 
controlling the reaction conditions and by reapplying the films, the shell thickness of the microcapsules can be tuned between $10 \mathrm{~nm}$ to over $100 \mathrm{~nm}$. Importantly, depending on the phenolic compound used, non-degradable or degradable capsules can be prepared, allowing for the release profiles of cargo to be specifically tuned. Similarly, the phenolic compound used also determines what further chemistries or modifications can be used to functionalize the capsules. The technical advancement of moving towards rapid, robust film deposition methods that phenolic compounds allow for is an important step toward realizing easy-to-prepare nanoengineered drug delivery vehicles with enhanced mechanical properties and stimuliresponsive abilities. As mussels use reversible metal-phenolic coordination for the self-healing of their feet, capsule shells with self-healing abilities could be developed in the near future for smart and adaptive systems, capable of releasing and encapsulating cargo in-situ depending on concentrations of trace ions. Films prepared from phenolics other than DA and TA are expected to expand the functionality and applicability of phenolic-based microcapsules in the biomedical field.

\section{ACKNOWLEDGMENT}

H.E. thanks the Japan Society for the Promotion of Science (JSPS) for a postdoctoral fellowship for research abroad. F.C. acknowledges the Australian Research Council for funding under the Australian Laureate Fellowship scheme (FL120100030). 
(a)
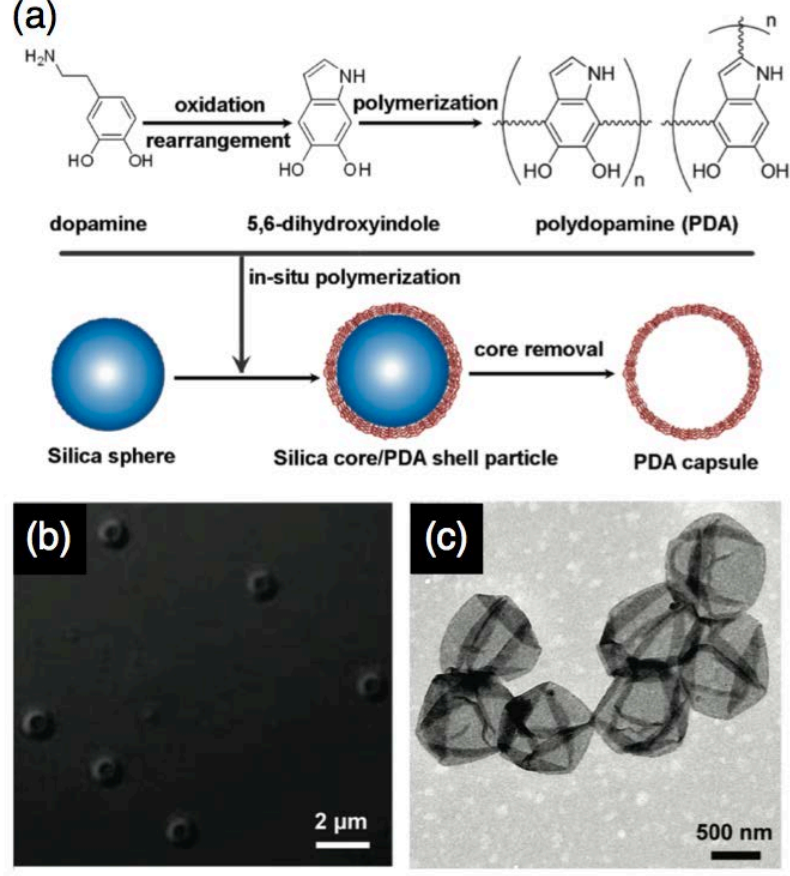

Figure 1. PDA film engineering for template-mediated capsule preparation. (a) Schematic illustration of the preparation process. (b,c) Differential interference contrast (DIC) microscopy (b) and transmission electron microscopy (TEM) (c) images of the PDA capsules. Reprinted with permission from ref ${ }^{23}$. Copyright 2009 American Chemical Society.

(a)

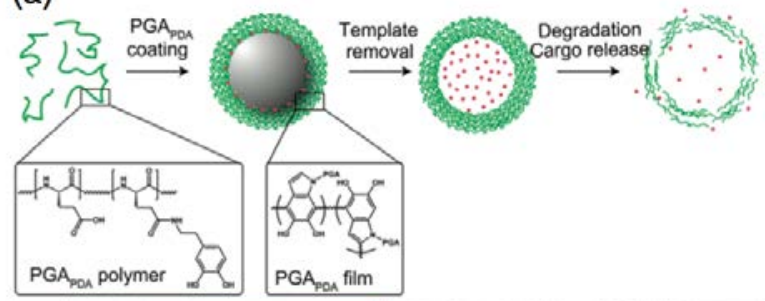

(b)

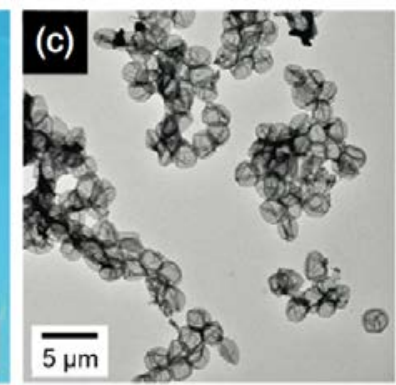

Figure 2. Film assembly of DA-modified PGA on particles for capsule preparation. (a) Schematic illustration of the preparation process. (b,c) DIC microscopy (b) and TEM (c) images of the capsules. Reprinted with permission from ref $^{26}$. Copyright 2011 American Chemical Society. 


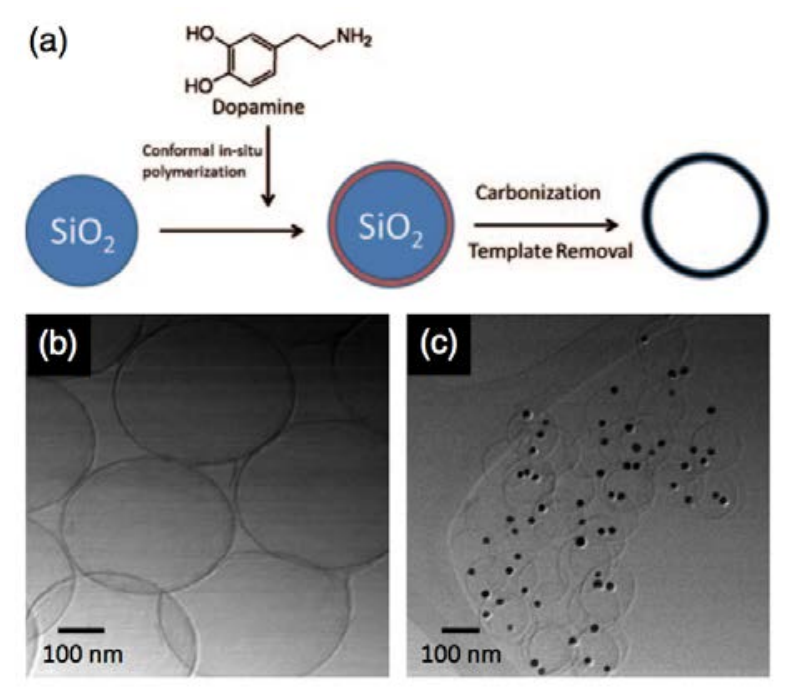

Figure 3. Carbon capsules prepared from the PDA precursor. (a) Schematic illustration of the preparation process. (b,c) Scanning TEM images of carbon capsules (b) and Au@C yolk-shell composites (c). Reprinted with permission from ref $^{27}$. Copyright 2011 WILEY-VCH Verlag GmbH \& Co. KGaA, Weinheim.

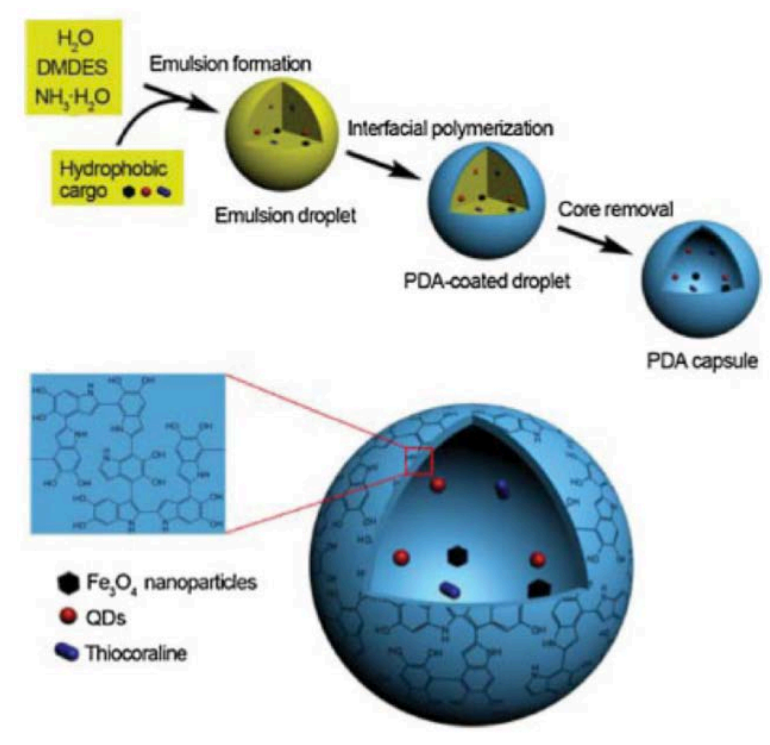

Figure 4. Schematic illustration of the PDA capsules prepared from an emulsion template and the loading of hydrophobic cargo. Reprinted with permission from ref $^{30}$. Copyright 2011 WILEY-VCH Verlag GmbH \& Co. KGaA, Weinheim. 


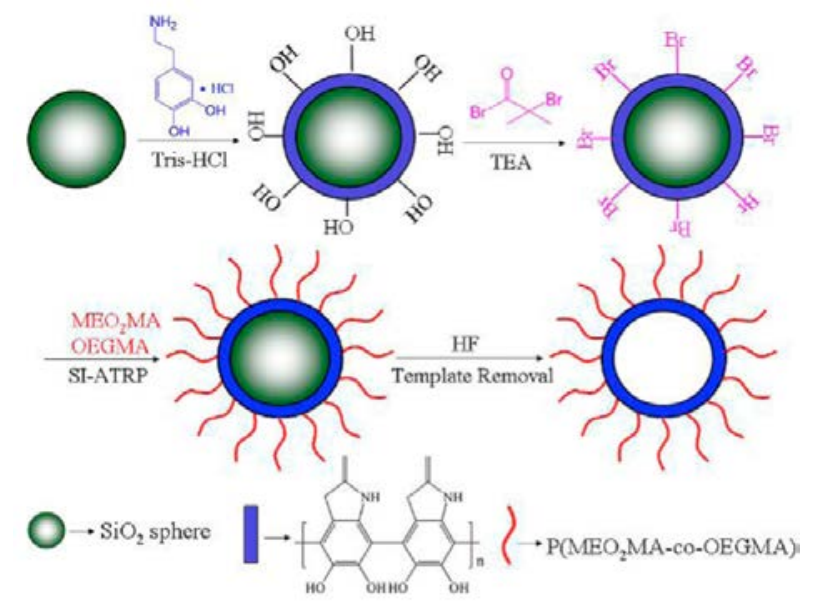

Figure 5. Schematic illustration of polymer grafting from PDA capsules via ATRP. Reprinted with permission from ref ${ }^{33}$. Copyright 2013 American Chemical Society.

(a)
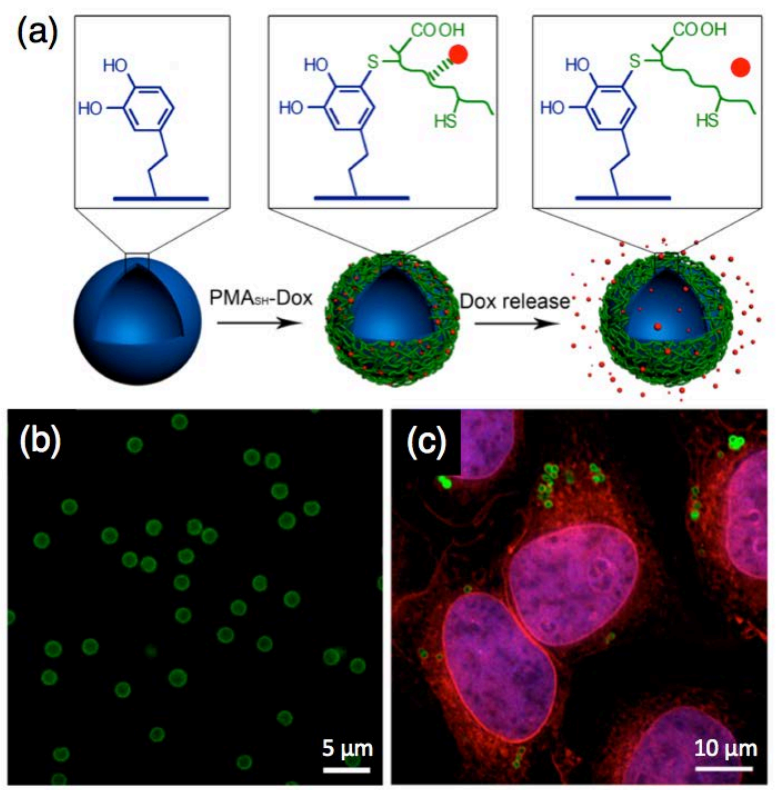

Figure 6. Drug delivery using PDA capsules. (a) Schematic illustration of the preparation of PMA $_{\mathrm{SH}^{-}}$-Dox loaded PDA capsules. (b) Fluorescence microscopy image of the Dox-loaded PDA capsules. (c) Deconvolution microscopy image of the uptake of the PDA capsules (green) by HeLa cells. Reprinted with permission from ref ${ }^{36}$. Copyright 2012 American Chemical Society. 


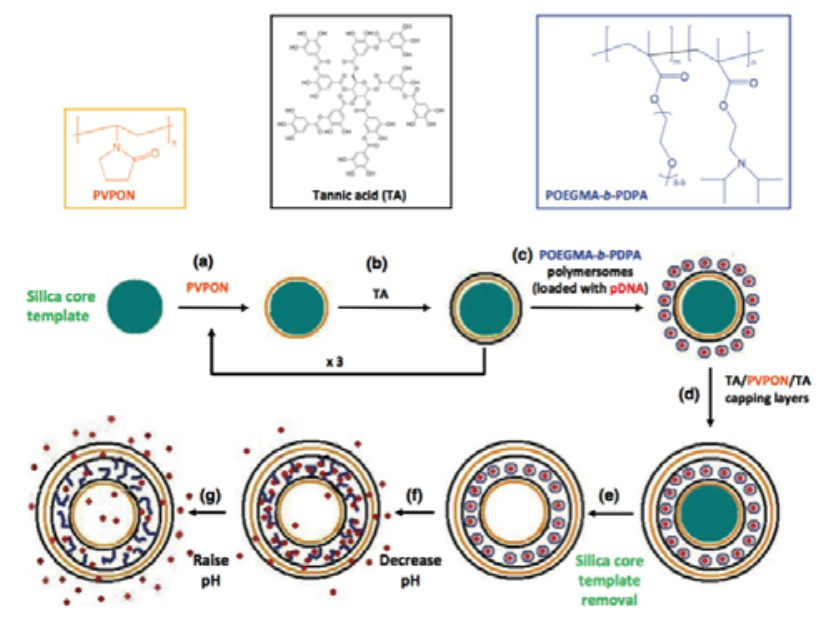

Figure 7. Schematic illustration of the preparation of polymersome-loaded LbL capsules. Reprinted with permission from ref ${ }^{52}$. Copyright 2011 WILEY-VCH Verlag GmbH \& Co. KGaA, Weinheim.

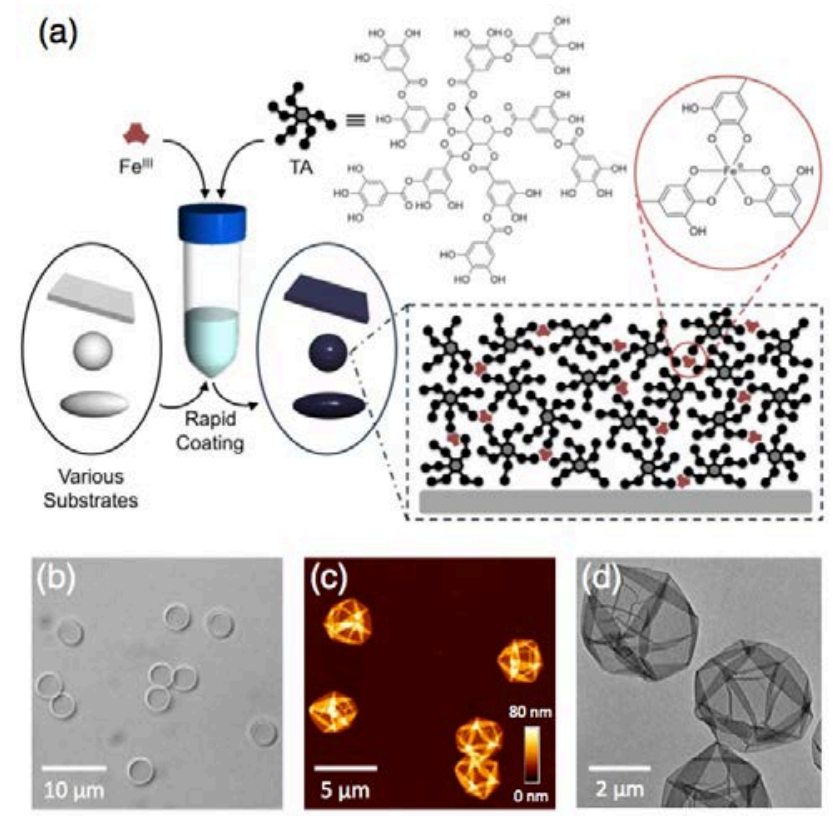

Figure 8. One-step assembly of $\mathrm{Fe}^{\mathrm{III}}-\mathrm{TA}$ films. (a) Schematic illustration of the Fe ${ }^{\mathrm{III}}-\mathrm{TA}$ film assembly. (b-d) DIC (b), AFM (c), TEM (d) images of $\mathrm{Fe}^{\mathrm{III}}$-TA capsules. Reprinted with permission from ref ${ }^{22}$. Copyright 2013 American Association for the Advancement of Science. 


\section{Author Profiles}

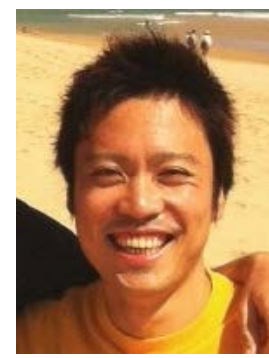

Hirotaka Ejima was born in Kanagawa Prefecture, Japan in 1983. He completed his Bachelor's degree under the supervision of Professor Yoshio Umezawa in 2006, his Master's degree under Professor Naoko Yoshie in 2008, and his PhD under Professor Takeshi Serizawa in 2011 at The University of Tokyo. He then joined the research group of Professor Frank Caruso at The University of Melbourne as a postdoctoral fellow. After spending two and a half years in Australia, he moved to be an assistant professor at the Institute of Industrial Science, The University of Tokyo. His current research interest is on developing functional nanomaterials based on renewable bioresources.

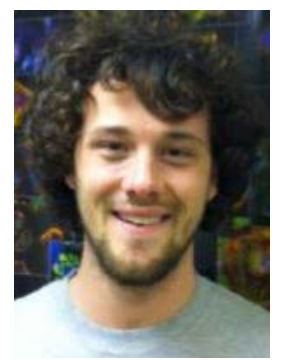

Joseph J. Richardson was born in the 'Sunshine State' Florida. He received his Bachelor's degree in Philosophy and his Master's in Industrial and Systems Engineering from the University of Florida. J.J. joined Professor Caruso's research group at The University of Melbourne as a postgraduate student in 2011. His $\mathrm{PhD}$ project is on the development of rapid, facile and automated microcapsule preparation systems.

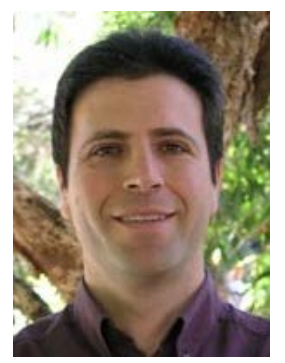

Frank Caruso is a professor and ARC Australian Laureate Fellow at The University of Melbourne. He received his PhD degree in 1994 from The University of Melbourne, and then moved to the CSIRO Division of Chemicals and Polymers in Melbourne. He was an Alexander von Humboldt Research Fellow and then group leader at the Max Planck Institute of Colloids and Interfaces from 1997-2002. His research interests focus on developing advanced nano- and biomaterials for biotechnology and medicine. He is the recipient of the inaugural 2012 ACS Nano Lectureship Award (Asia/Pacific) from the American Chemical Society. In 2012, he was 
awarded the Royal Society of Victoria Medal for Excellence in Scientific Research in the Physical Sciences, and in 2013 the CSIRO Eureka Prize for Leadership in Science. 


\section{References}

1 Cui, J., van Koeverden, M. P., Müllner, M., Kempe, K. \& Caruso, F. Emerging methods for the fabrication of polymer capsules. Adv. Colloid Interface Sci. in press DOI: 10.1016/j.cis.2013.10.012.

2 Discher, B. M., Won, Y. Y., Ege, D. S., Lee, J. C. M., Bates, F. S., Discher, D. E. \& Hammer, D. A. Polymersomes: tough vesicles made from diblock copolymers. Science 284, 1143-1146 (1999).

3 Kishimura, A. Development of polyion complex vesicles (PICsomes) from block copolymers for biomedical applications. Polymer J. 45, 892-897 (2013).

$4 \quad$ Wang, Y., Angelatos, A. S. \& Caruso, F. Template synthesis of nanostructured materials via layer-by-layer assembly. Chem. Mater. 20, 848-858 (2008).

5 Ariga, K., Hill, J. P. \& Ji, Q. Layer-by-layer assembly as a versatile bottom-up nanofabrication technique for exploratory research and realistic application. Phys. Chem. Chem. Phys. 9, 2319-2340 (2007).

6 Hammond, P. T. Building biomedical materials layer-by-layer. Mater. Today 15, 196206 (2012).

7 Decher, G. Fuzzy nanoassemblies: toward layered polymeric multicomposites. Science 277, 1232-1237 (1997).

8 Kondo, K., Kida, T., Ogawa, Y., Arikawa, Y. \& Akashi, M. Nanotube formation through the continuous one-dimensional fusion of hollow nanocapsules composed of layer-bylayer poly(lactic acid) stereocomplex films. J. Am. Chem. Soc. 132, 8236-8237 (2010).

9 Kida, T., Mouri, M. \& Akashi, M. Fabrication of hollow capsules composed of poly(methyl methacrylate) stereocomplex films. Angew. Chem. Int. Ed. 45, 7534-7536 (2006).

10 Ariga, K., Ji, Q. M., Hill, J. P., Bando, Y. \& Aono, M. Forming nanomaterials as layered functional structures toward materials nanoarchitectonics. NPG Asia Mater. 4, 11 (2012).

11 Yan, Y., Björnmalm, M. \& Caruso, F. Assembly of layer-by-layer particles and their interactions with biological systems. Chem. Mater. 26, 452-460 (2014).

12 Donath, E., Sukhorukov, G. B., Caruso, F., Davis, S. A. \& Möhwald, H. Novel hollow polymer shells by colloid-templated assembly of polyelectrolytes. Angew. Chem. Int. Ed. 37, 2202-2205 (1998).

13 Richardson, J. J., Ejima, H., Lörcher, S. L., Liang, K., Senn, P., Cui, J. \& Caruso, F. Preparation of nano- and microcapsules by electrophoretic polymer assembly. Angew. Chem. Int. Ed. 52, 6455-6458 (2013).

14 Richardson, J. J., Liang, K., Kempe, K., Ejima, H., Cui, J. \& Caruso, F. Immersive polymer assembly on immobilized particles for automated capsule preparation. Adv. Mater. 25, 6874-6878 (2013).

15 Bentley, W. E. \& Payne, G. F. Nature's other self-assemblers. Science 341, 136-137 (2013).

16 Quideau, S., Deffieux, D., Douat-Casassus, C. \& Pouysegu, L. Plant polyphenols: chemical properties, biological activities, and synthesis. Angew. Chem. Int. Ed. 50, 586621 (2011).

17 Waite, J. H. \& Tanzer, M. L. Polyphenolic substance of Mytilus edulis - novel adhesive containing L-dopa and hydroxyproline. Science 212, 1038-1040 (1981). 
18 Sileika, T. S., Barrett, D. G., Zhang, R., Lau, K. H. A. \& Messersmith, P. B. Colorless multifunctional coatings inspired by polyphenols found in tea, chocolate, and wine. Angew. Chem. Int. Ed. 52, 10766-10770 (2013).

19 Lee, H., Dellatore, S. M., Miller, W. M. \& Messersmith, P. B. Mussel-inspired surface chemistry for multifunctional coatings. Science 318, 426-430 (2007).

20 Dreyer, D. R., Miller, D. J., Freeman, B. D., Paul, D. R. \& Bielawski, C. W. Elucidating the structure of poly(dopamine). Langmuir 28, 6428-6435 (2012).

21 Hong, S., Na, Y. S., Choi, S., Song, I. T., Kim, W. Y. \& Lee, H. Non-covalent selfassembly and covalent polymerization co-contribute to polydopamine formation. Adv. Funct. Mater. 22, 4711-4717 (2012).

22 Ejima, H., Richardson, J. J., Liang, K., Best, J. P., van Koeverden, M. P., Such, G. K., Cui, J. \& Caruso, F. One-step assembly of coordination complexes for versatile film and particle engineering. Science 341, 154-157 (2013).

23 Postma, A., Yan, Y., Wang, Y., Zelikin, A. N., Tjipto, E. \& Caruso, F. Selfpolymerization of dopamine as a versatile and robust technique to prepare polymer capsules. Chem. Mater. 21, 3042-3044 (2009).

24 Yu, B., Wang, D. A., Ye, Q., Zhou, F. \& Liu, W. Robust polydopamine nano/microcapsules and their loading and release behavior. Chem. Commun. 6789-6791 (2009).

25 Liu, Q., Yu, B., Ye, W. \& Zhou, F. Highly selective uptake and release of charged molecules by pH-responsive polydopamine microcapsules. Macromol. Biosci. 11, 12271234 (2011).

26 Ochs, C. J., Hong, T., Such, G. K., Cui, J., Postma, A. \& Caruso, F. Dopamine-mediated continuous assembly of biodegradable capsules. Chem. Mater. 23, 3141-3143 (2011).

27 Liu, R., Mahurin, S. M., Li, C., Unocic, R. R., Idrobo, J. C., Gao, H. J., Pennycook, S. J. \& Dai, S. Dopamine as a carbon source: the controlled synthesis of hollow carbon spheres and yolk-structured carbon nanocomposites. Angew. Chem. Int. Ed. 50, 67996802 (2011).

28 Arnal, P. M., Comotti, M. \& Schüth, F. High-temperature-stable catalysts by hollow sphere encapsulation. Angew. Chem. Int. Ed. 45, 8224-8227 (2006).

29 Zoldesi, C. I. \& Imhof, A. Synthesis of monodisperse colloidal spheres, capsules, and microballoons by emulsion templating. Adv. Mater. 17, 924-928 (2005).

30 Cui, J., Wang, Y., Postma, A., Hao, J., Hosta-Rigau, L. \& Caruso, F. Monodisperse polymer capsules: tailoring size, shell thickness, and hydrophobic cargo loading via emulsion templating. Adv. Funct. Mater. 20, 1625-1631 (2010).

$31 \mathrm{Xu}, \mathrm{H}$., Liu, X. \& Wang, D. Interfacial basicity-guided formation of polydopamine hollow capsules in pristine $\mathrm{O} / \mathrm{W}$ emulsions - toward understanding of emulsion template roles. Chem. Mater. 23, 5105-5110 (2011).

32 Kohri, M., Kohma, H., Shinoda, Y., Yamauchi, M., Yagai, S., Kojima, T., Taniguchi, T. \& Kishikawa, K. A colorless functional polydopamine thin layer as a basis for polymer capsules. Polym. Chem. 4, 2696-2702 (2013).

33 Ma, Z., Jia, X., Hu, J., Zhang, G., Zhou, F., Liu, Z. \& Wang, H. Dual-responsive capsules with tunable low critical solution temperatures and their loading and release behavior. Langmuir 29, 5631-5637 (2013).

34 Zhang, L., Shi, J., Jiang, Z., Jiang, Y., Meng, R., Zhu, Y., Liang, Y. \& Zheng, Y. Facile preparation of robust microcapsules by manipulating metal-coordination interaction 
between biomineral layer and bioadhesive layer. ACS Appl. Mater. Interfaces 3, 597-605 (2011).

35 Wang, X., Jiang, Z., Shi, J., Liang, Y., Zhang, C. \& Wu, H. Metal-organic coordinationenabled layer-by-layer self-assembly to prepare hybrid microcapsules for efficient enzyme immobilization. ACS Appl. Mater. Interfaces 4, 3476-3483 (2012).

36 Cui, J., Yan, Y., Such, G. K., Liang, K., Ochs, C. J., Postma, A. \& Caruso, F. Immobilization and intracellular delivery of an anticancer drug using mussel-inspired polydopamine capsules. Biomacromolecules 13, 2225-2228 (2012).

37 Lopes, G. K. B., Schulman, H. M. \& Hermes-Lima, M. Polyphenol tannic acid inhibits hydroxyl radical formation from Fenton reaction by complexing ferrous ions. Biochim. Biophys. Acta 1472, 142-152 (1999).

38 Akiyama, H., Fujii, K., Yamasaki, O., Oono, T. \& Iwatsuki, K. Antibacterial action of several tannins against Staphylococcus aureus. J. Antimicrob. Chemother. 48, 487-491 (2001).

39 Athar, M., Khan, W. A. \& Mukhtar, H. Effect of dietary tannic acid on epidermal, lung, and forestomach polycyclic aromatic hydrocarbon metabolism and turmorigenicity in sencar mice. Cancer Res. 49, 5784-5788 (1989).

40 Chen, S. C. \& Chung, K. T. Mutagenicity and antimutagenicity studies of tannic acid and its related compounds. Food Chem. Toxicol. 38, 1-5 (2000).

41 Zhang, X. F., Dai, Y. C., Zhong, W., Tan, M., Lv, Z. P., Zhou, Y. C. \& Jiang, X. Tannic acid inhibited norovirus binding to HBGA receptors, a study of 50 Chinese medicinal herbs. Bioorg. Med. Chem. 20, 1616-1623 (2012).

42 Shutava, T., Prouty, M., Kommireddy, D. \& Lvov, Y. pH responsive decomposable layer-by-layer nanofilms and capsules on the basis of tannic acid. Macromolecules 38, 2850-2858 (2005).

43 Shutava, T. G., Prouty, M. D., Agabekov, V. E. \& Lvov, Y. M. Antioxidant properties of layer-by-layer films on the basis of tannic acid. Chem. Lett. 35, 1144-1145 (2006).

44 Shutova, T. G., Agabekov, V. E. \& Lvov, Y. M. Reaction of radical cations with multilayers of tannic acid and polyelectrolytes. Russ. J. Gen. Chem. 77, 1494-1501 (2007).

45 Erel-Unal, I. \& Sukhishvili, S. A. Hydrogen-bonded multilayers of a neutral polymer and a polyphenol. Macromolecules 41, 3962-3970 (2008).

46 Kozlovskaya, V., Kharlampieva, E., Drachuk, I., Cheng, D. \& Tsukruk, V. V. Responsive microcapsule reactors based on hydrogen-bonded tannic acid layer-by-layer assemblies.

Soft Matter 6, 3596-3608 (2010).

47 Lisunova, M. O., Drachuk, I., Shchepelina, O. A., Anderson, K. D. \& Tsukruk, V. V. Direct probing of micromechanical properties of hydrogen-bonded layer-by-layer microcapsule shells with different chemical compositions. Langmuir 27, 11157-11165 (2011).

48 Antunes, A. B. d. F., Dierendonck, M., Vancoillie, G., Remon, J. P., Hoogenboom, R. \& De Geest, B. G. Hydrogen bonded polymeric multilayer films assembled below and above the cloud point temperature. Chem. Commun. 49, 9663-9665 (2013).

49 Kim, S., Kim, D. S. \& Kang, S. M. Reversible layer-by-layer deposition on solid substrates inspired by mussel byssus cuticle. Chem. Asian J. 9, 63-66 (2014). 
50 Rahim, M. A., Ejima, H., Cho, K. L., Kempe, K., Müllner, M., Best, J. P. \& Caruso, F. Coordination-driven multistep assembly of metal-polyphenol films and capsules. Chem. Mater. 26, 1645-1653 (2014).

51 Shukla, A., Fang, J. C., Puranam, S., Jensen, F. R. \& Hammond, P. T. Hemostatic multilayer coatings. Adv. Mater. 24, 492-496 (2012).

52 Lomas, H., Johnston, A. P. R., Such, G. K., Zhu, Z., Liang, K., van Koeverden, M. P., Alongkornchotikul, S. \& Caruso, F. Polymersome-loaded capsules for controlled release of DNA. Small 7, 2109-2119 (2011).

53 Vinogradova, O. I., Lebedeva, O. V. \& Kim, B. S. Mechanical behavior and characterization of microcapsules. Annu. Rev. Mater. Res. 36, 143-178 (2006).

54 Guo, J., Ping, Y., Ejima, H., Alt, K., Meissner, M., Richardson, J. J., Yan, Y., Peter, K., Elverfeldt, D., Hagemeyer, C. E., \& Caruso, F. Engineering Multifunctional capsules through the assembly of metal-phenolic networks. Angew. Chem. Int. Ed. in press. DOI: http://dx.doi.org/10.1002/anie.201311136. 